\title{
Simulation of a winter circulation over India using a global spectral model
}

\author{
S K DASH and B CHAKRAPANI \\ Centre for Atmospheric Sciences, Indian Institute of Technology, New Delhi 110016, India \\ MS received 22 August 1988; revised 1 February 1989

\begin{abstract}
A primitive equation spectral model has been successfully integrated for five days starting with the initial data of 26 February 1982 . The geopotential heights and wind strengths arc well predicted up to day 3 . The 24-hour accumulated precipitation of the model reasonably agrees only up to two days, with the observed rainfall under the influence of western disturbance that prevailed over Afghanistan, Pakistan and north India till 3 March 1982. For one day global integration of the model, the CPU time requirement in Cyber 170/730 is about one hour.
\end{abstract}

Keywords. Triangular truncation; semi-implicit scheme; transform method; normal mode initialization; western disturbance.

\section{Introduction}

This paper deals with the prediction of a winter circulation over India in February 1982 by the Global Spectral Model (GSM) of IIT, Delhi. The original version of GSM belongs to the European Centre for Medium range Weather Forecasts (ECMWF). The modified L5T21 GSM has been successfully integrated globally for five days in Cyber 170/730 available at the National Informatics Centre, Delhi. At present the model has five sigma levels in the vertical and the horizontal resolution and is limited to 21 waves in the triangular truncation scheme. This paper briefly describes the dynamics of the GSM and the physical parametrization schemes before discussing the results of five-day-integrations. The initial date of integration is chosen to be 26 February 1982 because of some significant weather over Indian subcontinent during that week.

The results of integration are quite encouraging. Although the integrations have been done globally, the discussions in this paper are confined to the geopotential fields and isotachs at five pressure levels and precipitations. Predicted geopotential heights and wind strengths compare well with the respective analyses of ECMWF up to day 3. Because of limited space, some selected diagrams at $200 \mathrm{mb}$ and $700 \mathrm{mb}$ pressure levels are given in this paper. Root mean square errors and relative forecast errors of zonal and meridional wind components and geopotential heights are computed at all the five levels of the model. The relative forecast errors show reasonably good forecasts of geopotential and wind up to day 3. Since precipitation is the most important parameter of any numerical weather prediction model, we have also computed the model precipitation on all the five days starting from 27 February 1982. The 24-hour accumulated model precipitations compare well up to two days 
with the rainfall as reported in the Weekly Weather Report of IMD over north India under the influence of western disturbance.

\section{Dynamics of the model}

The mathematical formulation of the GSM is based on the equations for momentum, thermodynamics, mixing ratio, continuity and hydrostatic relation. The coordinate system chosen is spherical with the independent variables $\lambda$ (longitude), $\mu=\sin \phi$ ( $\phi$ being the latitude), $\sigma=p / p_{s}$ (Phillips 1957) and $t$ (time). Here $p_{s}$ denotes the surface pressure and $p$ the atmospheric pressure. Since the wind components on the globe are pseudoscalars (Robert 1966) dependent variables $U=u \cos \phi$ and $V=v \cos \phi$ are used instead of $u$ and $v$. The other dependent variables in the model are temperature $(T)$, mixing ratio $(q)$, surface pressure $\left(p_{s}\right)$ and some surface parameters like surface temperature, moisture and snow, soil temperature and moisture, surface roughness etc.

In the vertical plane the conventional finite difference approach similar to the formulation of Hoskins and Simmons (1975) and Burridge and Haseler (1977) has been adopted in the GSM. This vertical scheme conserves mass and energy but does not conserve angular mómentum.

In the horizontal plane, all prognostic quantities such as $\zeta$ (absolute vorticity), $D$ (divergence), $T, q, \ln p_{s}, \Phi$ (geopotential) and $\Phi_{s}$ (orography) are represented by truncated series of spherical harmonics of the form,

$$
X(\lambda, \mu, \sigma, t)=\sum_{M=-M}^{M} \sum_{n=|m|}^{N(m)} X_{n}^{m}(\sigma, t) P_{n}^{m}(\mu) \exp (\operatorname{im} \lambda),
$$

where $X_{n}^{m}$ are the complex spectral coefficients and $P_{n}^{m}$ is the associated Legendre function of the first kind of order $m$ and degree $n$. Here $M$ is the highest Fourier wave number included in the east-west representation and $N(m)$ is the highest degree of the associated Legendre function included in the north-south representation. As $m$ and $n$ increase, they correspond to features with decreasing horizontal scales.

In GSM, the truncation scheme introduced is the general pentagonal truncation. Because of this provision there is choice in choosing any of the special truncation schemes such as the rhomboidal, triangular or trapezoidal. For the present simulation we have used the triangular truncation scheme because of its advantages over other schemes. A theoretical advantage of the triangular scheme is that it is isotropic i.e., the resolution is uniform on the sphere. In the triangular truncation, $N(m)=M$ in series (1). We have truncated the series at $N=21$.

For time integration the semi-implicit scheme of Robert (1969) is used in the model. This scheme permits a time step of approximately six times the value acceptable in a fully explicit approach. This scheme is also convenient and efficient to be adopted in multilevel spectral model (Robert et al 1972). In this scheme the temperature (T) is separated into two parts:

$$
T^{\prime}(\lambda, \mu, \sigma, t)=T(\lambda, \mu, \sigma, t)-T_{0}(\sigma) .
$$

The basic temperature $T_{0}$ depends only on the vertical coordinate and $T^{\prime}$ is the perturbation temperature.

The momentum equations involving $u$ and $v$ are not convenient to be directly used 
in the spectral model (Bourke 1972). Hence the vorticity and divergence counterparts of the momentum equations are used in the GSM. After the introduction of the vertical finite difference scheme, the horizontal spectral representation and the semi-implicit scheme, the complete set of prognostic equations can be derived. Those equations are given below.

Vorticity equation:

$$
\delta_{t} \zeta_{n}^{m} \downarrow=Z_{n}^{m} \downarrow
$$

Thermodynamic equation:

$$
\delta_{t} T_{n}^{1 m} \downarrow=\Upsilon_{n}^{m} \downarrow-\tau \bar{D}_{n}^{t m} \downarrow
$$

Mixing ratio equation:

$$
\delta_{t} q_{n}^{m} \downarrow=Q_{n}^{m} \downarrow .
$$

Surface pressure tendency equation:

$$
\delta_{t} \ln p_{s n}^{m}=\mathscr{P}_{n}^{m}-\Pi \bar{D}_{n}^{t m} \downarrow
$$

Divergence equation:

and

$$
\begin{aligned}
\mathbf{A} n \bar{D}_{n}^{\prime m} \downarrow= & \frac{a^{2}}{n(n+1)} D_{n}^{m}(t-\Delta t) \downarrow \\
& +\Delta t\left(\frac{a^{2}}{n(n+1)} \mathscr{D}_{n}^{m} \downarrow+\mathscr{R}_{n}^{m} \downarrow+\Delta t R \mathbf{B} \Upsilon_{1}{ }_{n}^{m} \downarrow\right)
\end{aligned}
$$

$$
\delta_{t} D_{n}^{m} \downarrow=\frac{1}{\Delta t}\left[\bar{D}_{n}^{t m} \downarrow-D_{n}^{m}(t-\Delta t) \downarrow\right] .
$$

The actual form of the terms $Z, \mathscr{D}, \mathbf{Y}, \mathscr{P}, Q, \mathscr{R}$ and the matrices $\mathbf{B}, \mathbf{A} n, \tau$ and $\Pi$ are given in the appendix.

In the above equations, the vertical arrows indicate column vectors and the horizontal arrows row vectors. The following standard notations at discrete times are utilized while writing the equations.

$$
\begin{aligned}
\delta_{t} X & =\frac{\partial X}{\partial \tau}=\frac{X(t+\Delta t)-X(t-\Delta t)}{2 \Delta t} \\
\bar{X}^{t} & =\frac{1}{2} X(t+\Delta t)+X(t-\Delta t) .
\end{aligned}
$$

Here $\Delta t$ is the time step for integration and the notation $-t$ denotes the average value at time $t$.

In order to avoid the growth of the computational mode a linear time filter of Robert (1966) is applied to the prognostic variables. In GSM the computation during each time step is performed in two loops while scanning the latitudes from the poles towards the equator. As discussed in Baede et al (1979) this structure reduces the core requirement at the expense of input/output of grid point data and some extra grid point buffers in core. 
The model starts from grid point values at time $t$ and produces a forecast of the grid point values at a future time $(t+\Delta t)$ with the intermediate stage for computations in spectral space. First of all, the computations of nonlinear terms including physical parametrizations are completed at transform grids of the model at time $t$ and transformation from grid point to spectral space is done with the help of Fast Fourier Transforms (FFT) and Gaussian quadratures. The semi-implicit equations are solved in the spectral space and the spectral coefficients of the prognostic variables are computed at time $(t+\Delta t)$. Finally, the reverse transformation is done from spectral domain to the transform grids.

\section{Physical parametrization}

The general problem of relating the physical processes, such as convection and precipitation, clouds and radiation, and turbulent fluxes of heat, moisture and momentum that are unresolvable by numerical weather prediction models to the parameters resolved and predicted by the model is known as parametrization. Physical processes in GSM include dry and moist adiabatic adjustment, large scale condensation, radiation and fluxes of heat, moisture and momentum based on the GFDL (Geophysical Fluid Dynamics Laboratory) model (Smagorinsky et al 1965; Manabe et al 1965).

The parametrization of the transport processes to smaller unresolved scales in the horizontal plane is done through horizontal diffusion. We choose a linear $\nabla^{4}$ horizontal diffusion of vorticity, divergence, temperature and moisture on sigma surfaces of the model. Since these processes are assumed to be linear, their contributions are easily calculated in spectral space and are added as correction terms to the respective prognostic variables after completion of the adiabatic time step.

The vertical diffusion of momentum and moisture is also assumed to be linear. Above the surface layer the upward fluxes of momentum and moisture due to turbulent motions are given by,

and

$$
\begin{aligned}
\tau_{\lambda} & =-\rho K(\partial u / \partial z), \\
\tau_{\mu} & =-\rho K(\partial v / \partial z),
\end{aligned}
$$

$$
R=-\rho K(\partial q / \partial z)
$$

where $K=l^{2}(\partial \mathbf{v} / \partial z)$ and $l$ is the mixing length.

At the surface layer the fluxes are given in terms of bulk aerodynamic parametrization as in Smagorinsky (1963) and Holloway and Manabe (1971). The surface boundary layer is assumed to be at a height of $75 \mathrm{~m}$ and above $2.5 \mathrm{~km}$ the vorticity, divergence and moisture are unaffected by diffusion since the mixing length is assumed to be zero.

Over land, snow and sea ice the surface temperature is computed from an instantaneous surface energy balance with the assumption that the heat capacity of the earth's surface is zero. The earth's surface is assumed to be wet everywhere. Over open ocean the surface temperature is specified as climatological values. The distributions of land, snow, sea ice and ocean are prescribed and fixed during the model forecast.

The dry and moist adjustment scheme of Manabe et al (1965) is applied to create 
mutually adjusted temperature and moisture fields. If the predicted atmosphere is not saturated and the lapse rate exceeds the dry adiabatic lapse rate (unstable) the temperatures are modified to give a dry adiabatic lapse rate. If the atmosphere is saturated and the lapse rate exceeds the moist adiabatic lapse rate (unstable) the moisture and temperature fields are adjusted so that the atmosphere is just saturated and satisfies the moist adiabatic lapse rate. If the atmosphere is stable but saturated, the moisture field is adjusted to the saturated value and the temperature field is simultaneously adjusted to reflect the heating due to the release of latent heat. The excess of moisture is assumed to go into the precipitation. It is assumed that the liquid water storage capacity of the atmosphere is zero. The precipitations in the model are accumulated over every time step.

The change in temperature due to radiative transfer is computed as a function of the vertical distribution of atmospheric absorbers as well as of temperature (Manabe and Strickler 1964). The atmospheric absorbers which are taken into consideration are water vapour, carbon dioxide, ozone and clouds. The climatological distribution of water vapour as well as that of carbon dioxide and ozone is used. It is also assumed that the zenith angle of the Sun is constant with time. In the present simulation the radiation is called twice a day.

\section{Integration of the model}

The data set for integration of GSM is obtained from the operational analyses produced and archived in the ECMWF. We have selected 26 February 1982 as the starting day of integration because of significant cyclonic systems over the Indian subcontinent from 27 February 1982 till 3 March. There was widespread rain over north India during this period and the weather over the region was largely influenced by Western disturbances.

GSM has been installed and executed in the Cyber 170/730 computer located at the National Informatics Centre, New Delhi. The model has been integrated for five days. Because of limitations in the CPU-memory, the model at present has five levels in the vertical and triangular truncation scheme with 21 waves in the horizontal plane being chosen for the horizontal spectral representation of the prognostic variables. The input data set consists of temperature, zonal and meridional components of wind and relative humidity at five prescribed sigma levels of the model and the surface pressure and orography. The climatological mean values of surface parameters like snow cover, surface temperature, surface moisture, albedo, soil temperature, soil moisture, emissivity and roughness parameter are also provided as input data for the model.

In GSM there are 32 gaussian latitudes and 64 points along each latitude. In the model the computations are done latitude-wise from the poles towards the equator. CPU takes about 5 hours for 120 hours integration of the model. The domain of integration is global and the duration of each time step is 40 minutes.

The wind fields, temperature and moisture at the five sigma levels of the model and precipitation are forecasted by GSM. The geopotential heights at the model levels are computed by using the predicted temperatures. The values at the sigma levels are interpolated to five pressure levels such as $100 \mathrm{mb}, 200 \mathrm{mb}, 500 \mathrm{mb}, 700 \mathrm{mb}$ and $850 \mathrm{mb}$. 


\section{Results and discussion}

After post-processing the model output, the wind fields, temperature, moisture and geopotential heights at five pressure levels and the precipitation are obtained on all the five forecast days starting from 27 February 1982 till 3 March 1982 . We have included a limited number of diagrams relevant to the discussion. The initial geopotential heights and wind fields at $200 \mathrm{mb}$ and $700 \mathrm{mb}$ pressure levels are shown in figures 1 and 2. These are obtained from the operational analyses of ECMWF and taken in the GSM as input. The isotachs (figures 2) show that there was a strong westerly current in middle and upper troposphere covering the entire region from Africa up to north India. A trough is also visible (figure 1a) in the upper troposphere. These initial weather conditions are fed into the model and the forecast equations are integrated in order to get the forecasts for the next five days.

The predicted geopotential heights on 27 February (day 1), 1 March (day 3) and
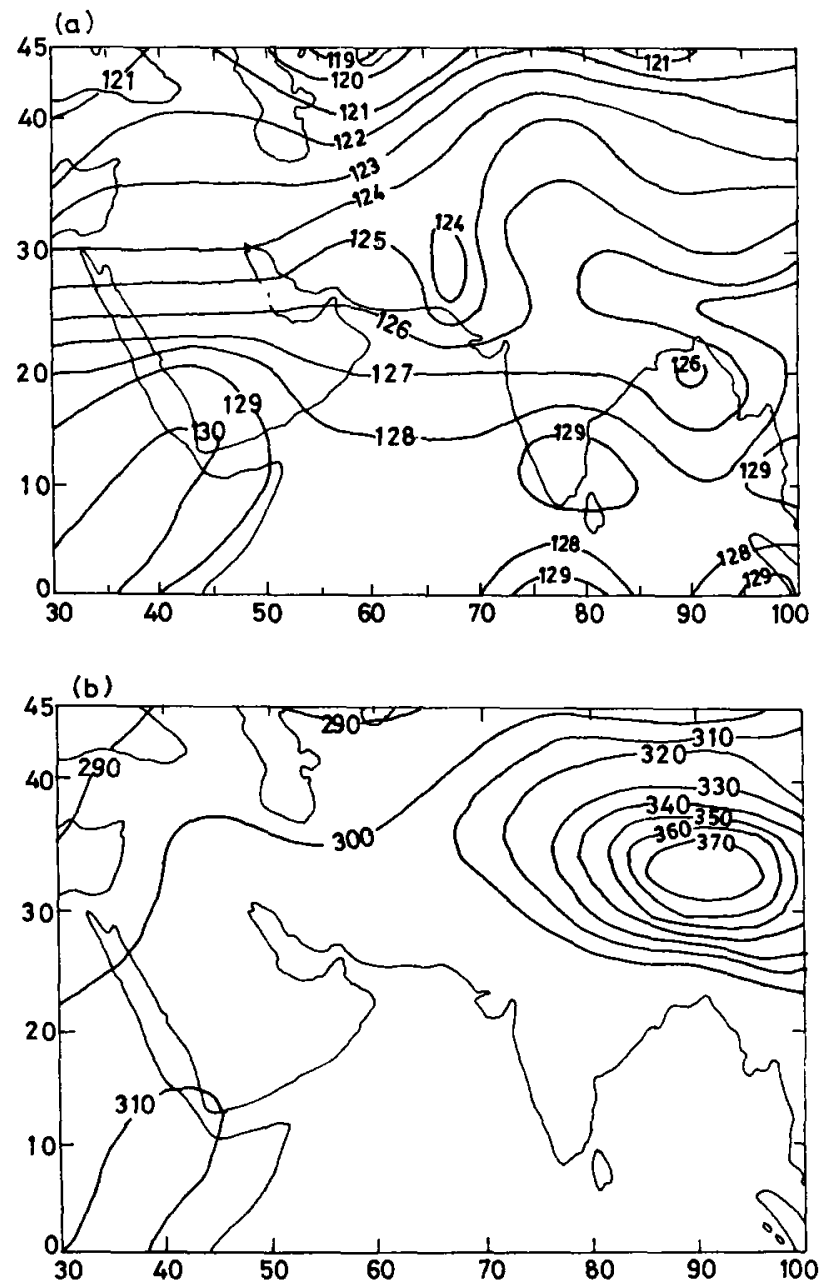

Figure 1. Geopotential analysis on 26.2 .1982 at (a) $200 \mathrm{mb}$ (in $10^{3} \mathrm{~m}^{2} \mathrm{~s}^{-2}$ ) and (b) $700 \mathrm{mb}$ (in $10^{2} \mathrm{~m}^{2} \mathrm{~s}^{-2}$ ) 

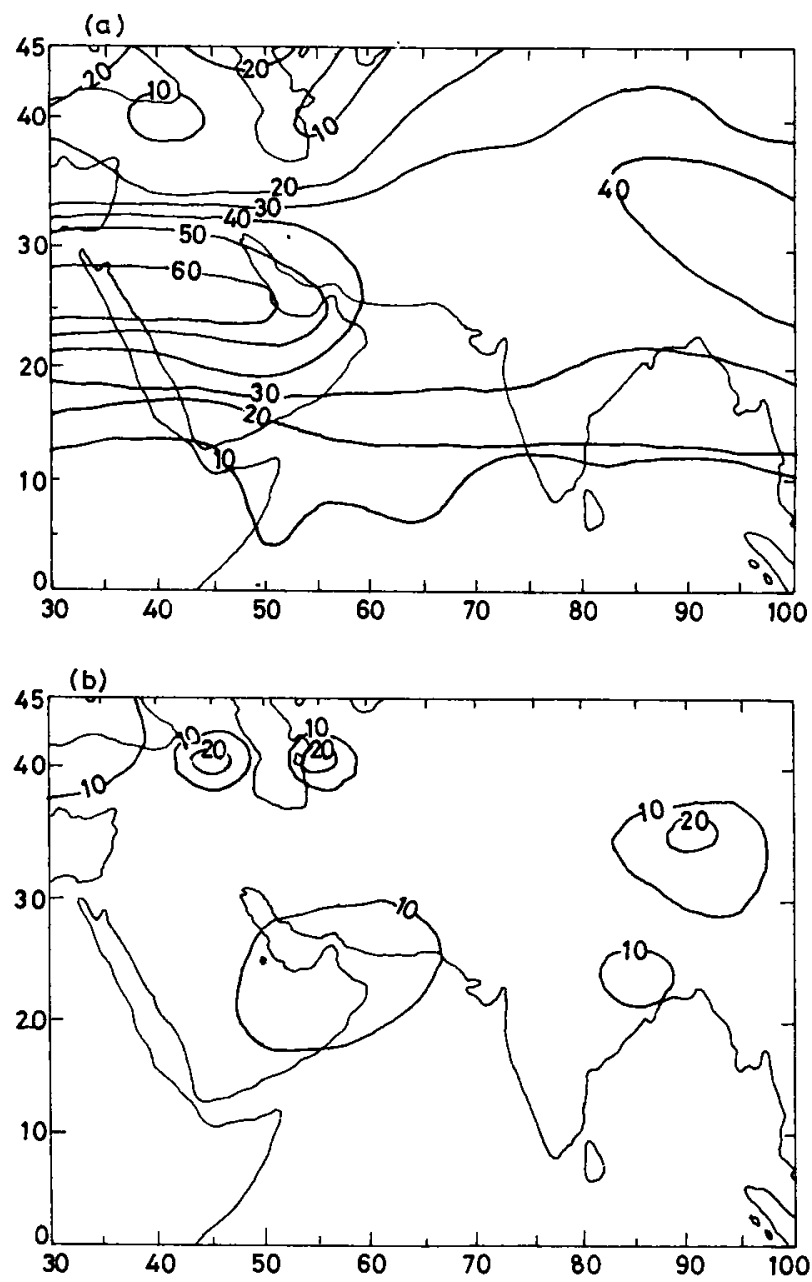

Figure 2. Isotachs $\left(\mathrm{m} \mathrm{s}^{-1}\right.$ ) analysis on 26.2 .1982 at (a) $200 \mathrm{mb}$ and (b) $700 \mathrm{mb}$.

3 March (day 5) at $200 \mathrm{mb}$ and $700 \mathrm{mb}$ pressure levels are shown in figures 3a, 4a, 5 and 6. For comparison, the analysed geopotentials of ECMWF on 27 February are also shown in figures $3 \mathrm{~b}$ and $4 \mathrm{~b}$. Comparison of predicted geopotential with the respective analyses on all the five days shows that the model geopotential heights are qualitatively good at both the levels up to day 3. The root mean square errors (RMSE) and the relative forecast errors (RFE) in the geopotential heights at the five levels over the region covered in the diagrams are given in tables 1 and 2 respectively. RFE is defined as the ratio of the standard deviation of forecast to that of persistance. The forecast may be treated as good or bad depending on whether RFE is less than one or greater than one respectively. RMSE are reasonable up to day 5 at all the levels except at $100 \mathrm{mb}$. RFE is less than one up to day 5, but the results are not good in the lower troposphere specially at $850 \mathrm{mb}$.

The forecast wind fields on days 1,3 and 5 are compared with the respective analyses of ECMWF and it is found that the forecast is qualitatively good up to day 3. 

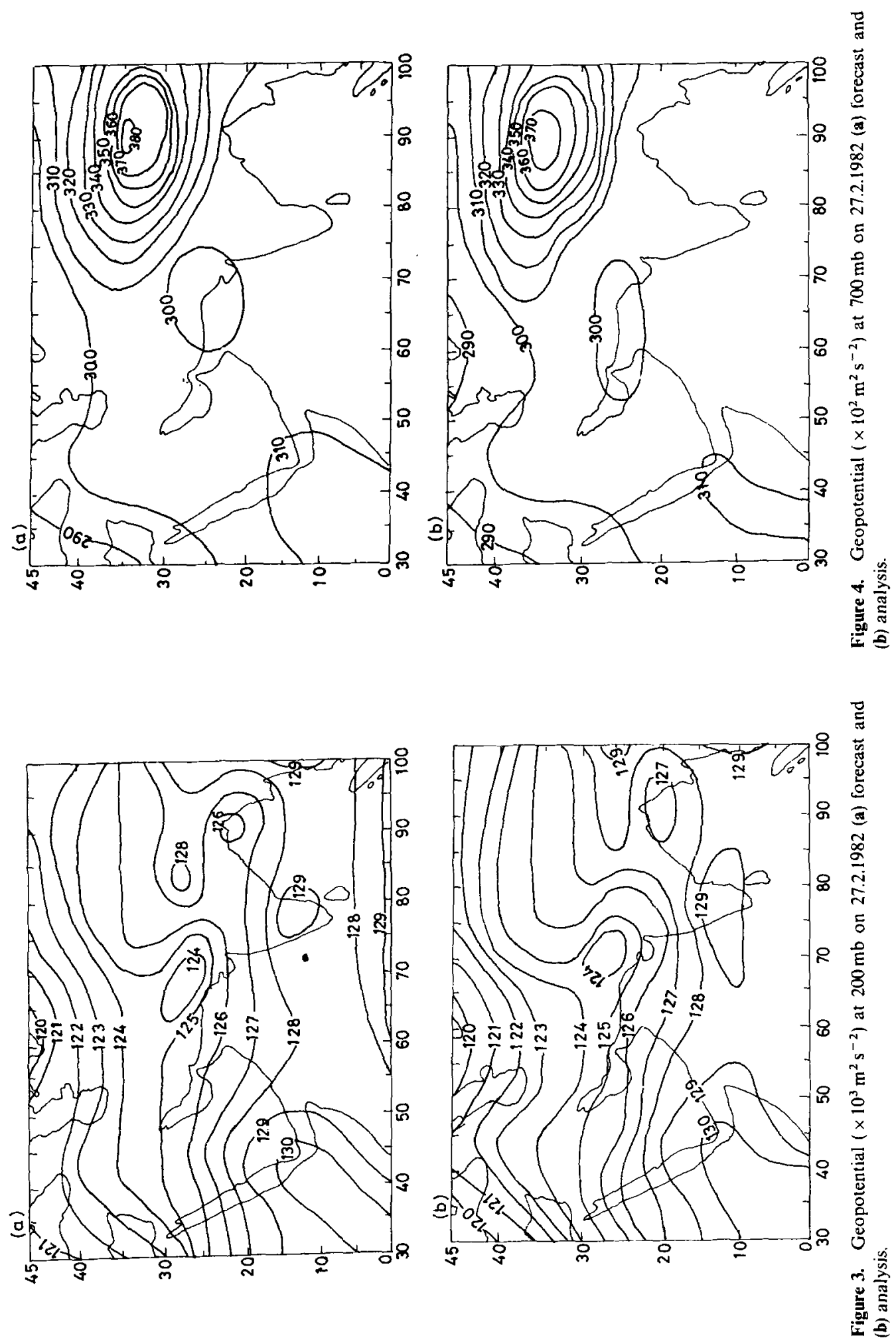

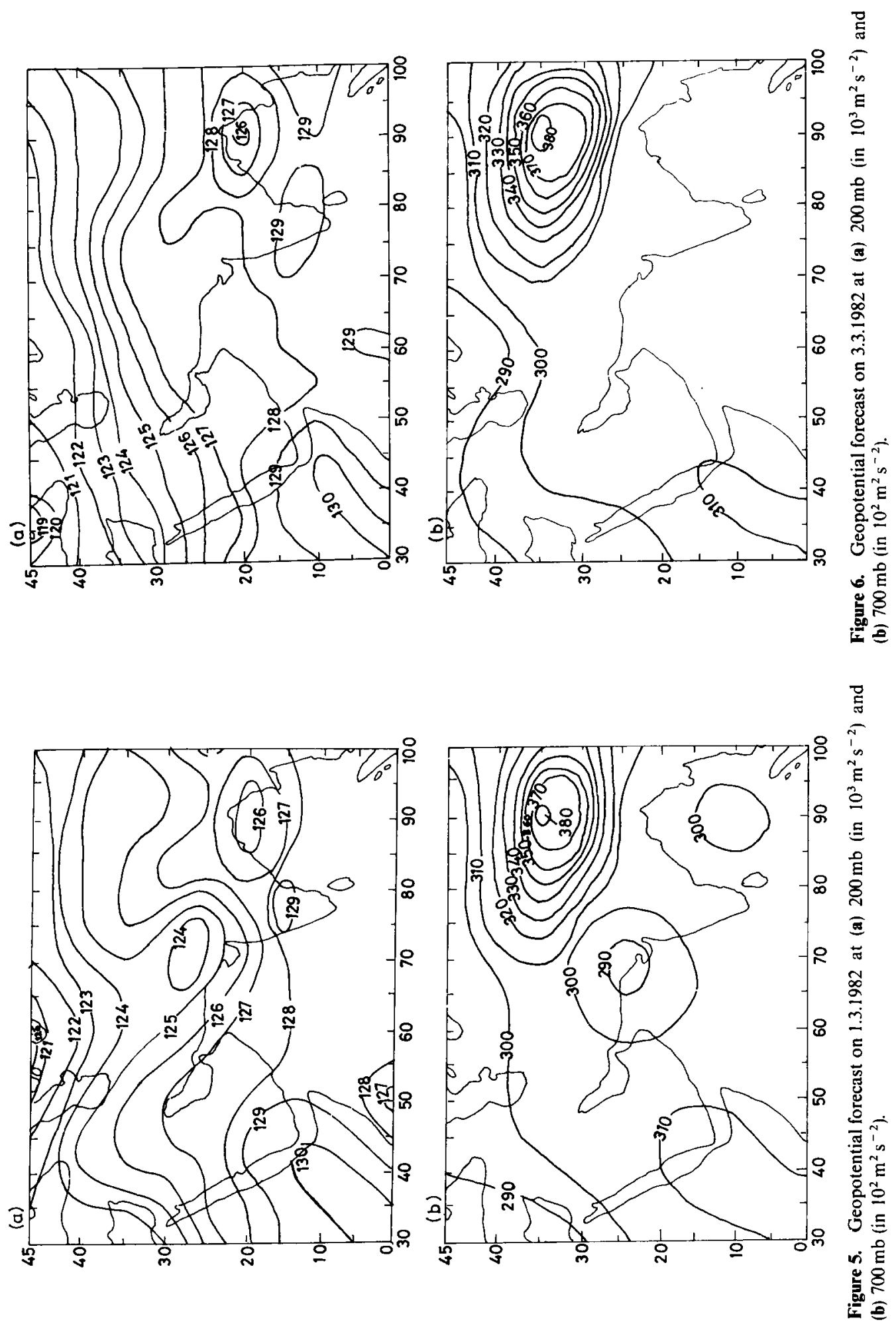
The forecasted isotachs on day 3 at $200 \mathrm{mb}$ and $700 \mathrm{mb}$ are shown in figure 7 . The RMSE and RFE of zonal $(u)$ and meridional $(v)$ components of wind are given in tables 1 and 2. The RFE of the meridional wind is less than one or comparable with one up to day 5 except at $850 \mathrm{mb}$. The forecast of zonal component of wind is good only up to day 2 below $500 \mathrm{mb}$. The RMSE of $u$ and $v$ are on the higher side. In general the quantitative forecast of winds is not good. It is found that the computed winds are underestimated.

Table 1. Root mean square errors of forecast fields ( $u$ and $v$ are expressed in $\mathrm{ms}^{-1}$ and $\Phi$ is in $\mathrm{m}^{2} \mathrm{~s}^{-2}$ ).

\begin{tabular}{|c|c|c|c|c|c|c|}
\hline $\begin{array}{l}\text { Forecast } \\
\text { day }\end{array}$ & Fields & $100 \mathrm{mb}$ & $200 \mathrm{mb}$ & $500 \mathrm{mb}$ & $700 \mathrm{mb}$ & $850 \mathrm{mb}$ \\
\hline \multirow{3}{*}{ Day 1} & $u$ & $6 \cdot 28$ & $6 \cdot 72$ & $3 \cdot 37$ & $3 \cdot 30$ & $4 \cdot 38$ \\
\hline & $v$ & 5.29 & $6 \cdot 15$ & $5 \cdot 22$ & $5 \cdot 63$ & 13.59 \\
\hline & $\Phi$ & $599 \cdot 34$ & 387.73 & $512 \cdot 26$ & $419 \cdot 86$ & $517 \cdot 69$ \\
\hline \multirow[t]{3}{*}{ Day 2} & $u$ & $7 \cdot 78$ & 7.91 & $6 \cdot 34$ & 5.95 & 11.87 \\
\hline & $v$ & $5 \cdot 53$ & $7 \cdot 81$ & $7 \cdot 44$ & $5 \cdot 71$ & 8.52 \\
\hline & $\Phi$ & $805 \cdot 17$ & $522 \cdot 56$ & $483 \cdot 70$ & $394 \cdot 35$ & $545 \cdot 19$ \\
\hline \multirow[t]{3}{*}{ Day 3} & $u$ & $9 \cdot 94$ & $12 \cdot 56$ & 9.06 & $7 \cdot 32$ & 14.81 \\
\hline & $v$ & $10 \cdot 34$ & 12.88 & 9.50 & $6 \cdot 84$ & 7.98 \\
\hline & $\Phi$ & $941 \cdot 70$ & $658 \cdot 14$ & 392.95 & $277 \cdot 19$ & $444 \cdot 89$ \\
\hline \multirow[t]{3}{*}{ Day 4} & $u$ & $11 \cdot 16$ & 11.82 & $9 \cdot 37$ & $11 \cdot 64$ & $29 \cdot 31$ \\
\hline & $v$ & 14.81 & $14 \cdot 80$ & 8.75 & 8.48 & 19.79 \\
\hline & $\Phi$ & 997.44 & $778 \cdot 11$ & $271 \cdot 38$ & $239 \cdot 26$ & $437 \cdot 17$ \\
\hline \multirow[t]{3}{*}{ Day 5} & $u$ & $12 \cdot 65$ & $18 \cdot 06$ & $11 \cdot 42$ & $11 \cdot 25$ & $22-45$ \\
\hline & $v$ & $14 \cdot 75$ & $18 \cdot 35$ & 8.83 & $4 \cdot 70$ & 6.01 \\
\hline & $\Phi$ & $754 \cdot 85$ & $656 \cdot 03$ & 409.65 & 283.01 & $546 \cdot 04$ \\
\hline
\end{tabular}

Table 2. Relative forecast errors of predicted fields.

\begin{tabular}{lllllll}
\hline $\begin{array}{llllll}\text { Forecast } \\
\text { day }\end{array}$ & Fields & $100 \mathrm{mb}$ & $200 \mathrm{mb}$ & $500 \mathrm{mb}$ & $700 \mathrm{mb}$ & $850 \mathrm{mb}$ \\
\hline Day 1 & $u$ & 1.19 & 1.01 & 1.01 & 1.37 & 1.64 \\
& $v$ & 0.73 & 0.71 & 1.03 & 1.72 & 3.31 \\
& $\Phi$ & 1.25 & 1.01 & 1.28 & 2.17 & 5.33 \\
Day 2 & $u$ & 1.17 & 0.96 & 1.21 & 1.68 & 4.22 \\
& $v$ & 0.88 & 0.78 & 1.04 & 1.07 & 1.68 \\
& $\Phi$ & 1.18 & 0.95 & 1.13 & 1.77 & 3.28 \\
Day 3 & $u$ & 1.28 & 1.27 & 1.48 & 1.51 & 2.13 \\
& $v$ & 1.04 & 0.95 & 1.02 & 1.10 & 1.27 \\
& $\Phi$ & 0.90 & 0.73 & 0.75 & 1.01 & 2.62 \\
Day 4 & $u$ & 1.50 & 1.35 & 1.33 & 2.07 & 4.01 \\
& $v$ & 1.31 & 0.83 & 0.84 & 1.33 & 2.36 \\
& $\Phi$ & 0.84 & 0.84 & 0.71 & 1.32 & 2.85 \\
Day 5 & $u$ & 2.15 & 1.69 & 1.28 & 2.40 & 3.89 \\
& $v$ & 1.39 & 1.11 & 1.02 & 1.11 & 1.77 \\
& $\Phi$ & 0.78 & 0.83 & 1.14 & 1.74 & 4.75 \\
\hline
\end{tabular}



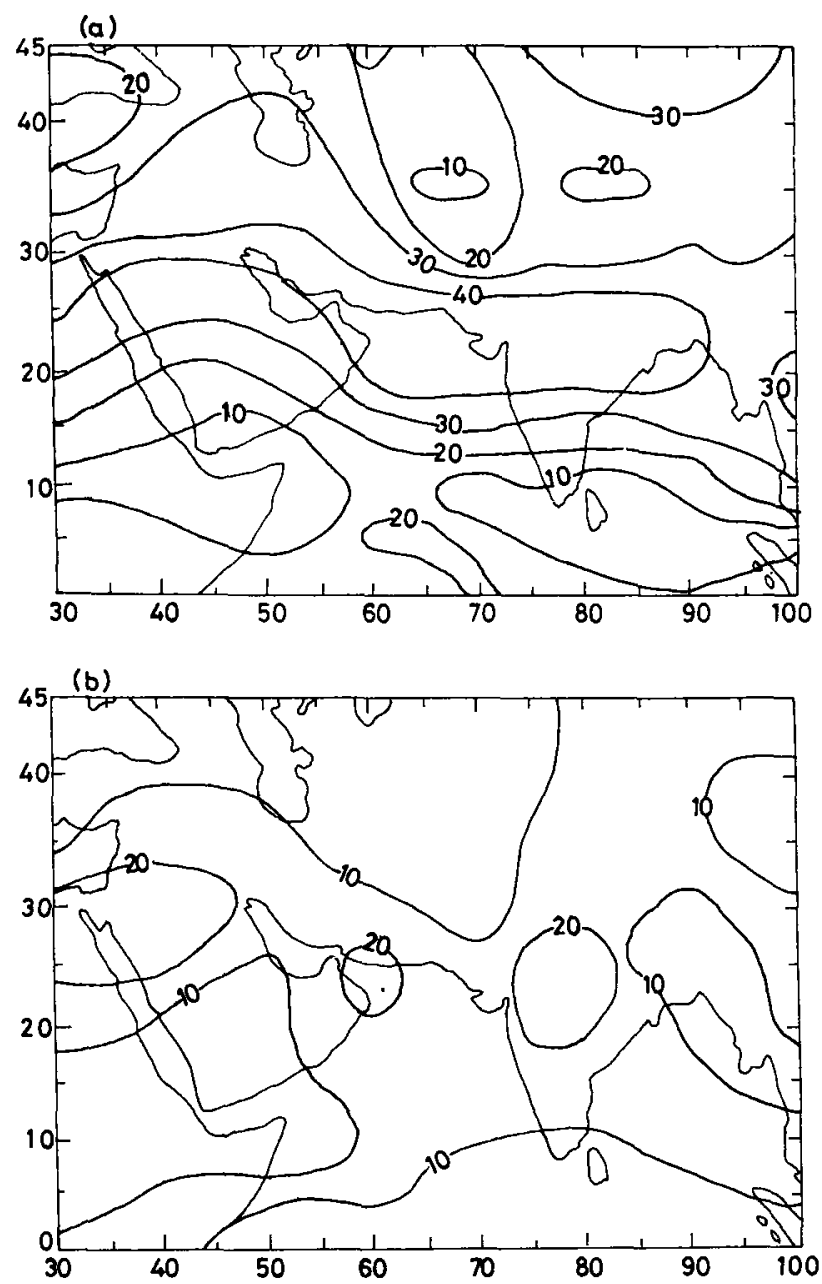

Figure 7. Isotachs $\left(\mathrm{m} \mathrm{s}^{-1}\right)$ forecast on 1.3 .1982 at (a) $200 \mathrm{mb}$ and (b) $700 \mathrm{mb}$.

The forecasts up to day 5 show that cyclonic circulations in the middle and upper tropospheric westerlies which lay over Afghanistan, Pakistan and North India on 26 February 1982 moved eastwards and became unimportant after 3 March 1982. These forecasts reasonably agree with the synoptic features over the region as reported in Weekly Weather Report by IMD. Under the influence of western disturbances there was widespread rain over north India. The 24-hour accumulated precipitation in the model from 27 February to 3 March 1982 is shown in figure 8. The observed rainfall during 24 hours as reported in the Indian Daily Weather Report by IMD is given in figure 9. Comparison of model precipitation with actual rainfall shows that there is qualitative agreement between the forecasted precipitation and the observed rainfall over north India. However, over north bay of Bengal and Arabian sea on days 4 and 5 there are some heavy spurious rainfall. Also on all the days over north India the rainfall is overpredicted by the model.

It may be noted that the horizontal resolution of the model is about $600 \mathrm{~km}$ and there are only five levels in the vertical. Hence the resolution of the model is not 

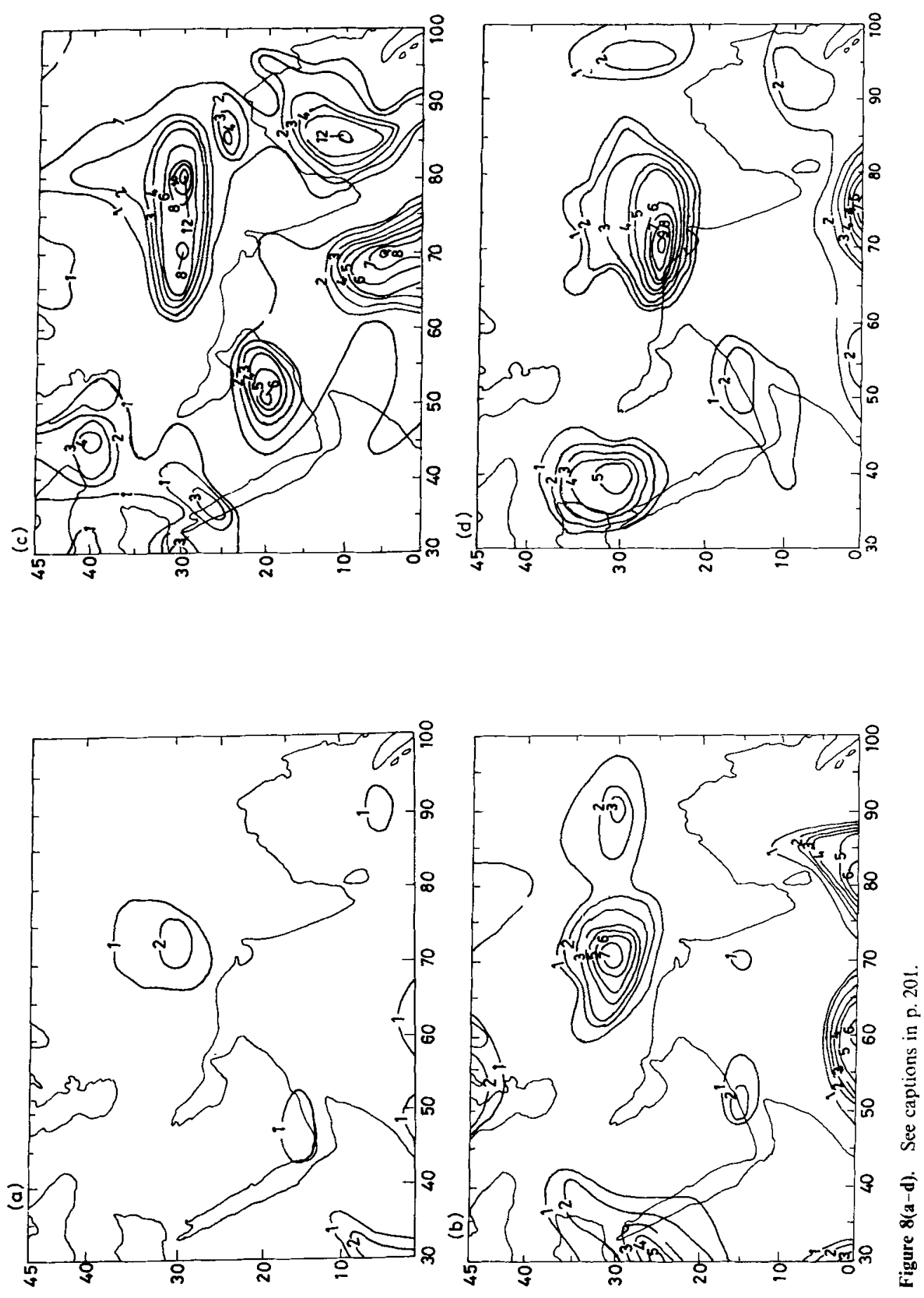


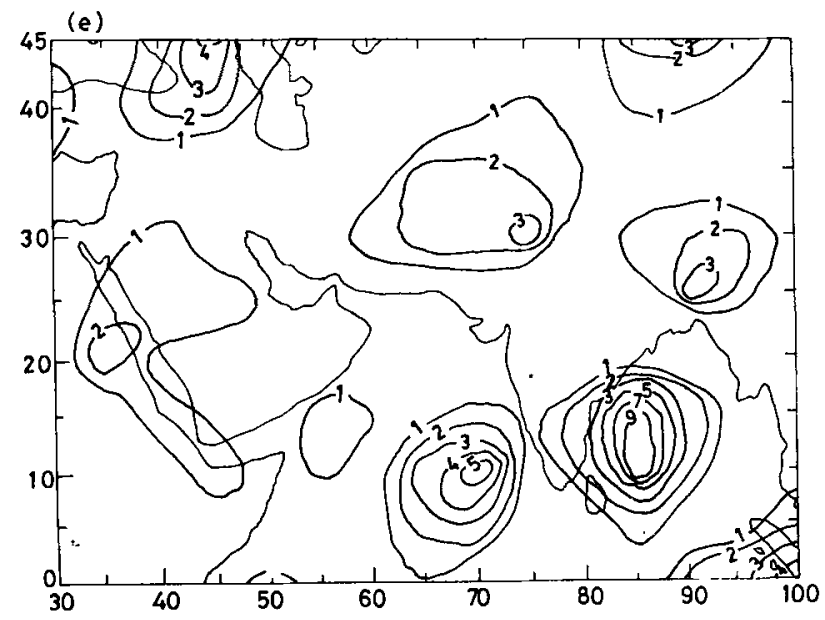

Figure 8. 24 hours accumulated precipitation (cm) forecast on (a) 27.2 .1982 (b) 28.2 .1982 (c) 1.3 .1982 (d) 2.3.1982 and (e) 3.3.1982.
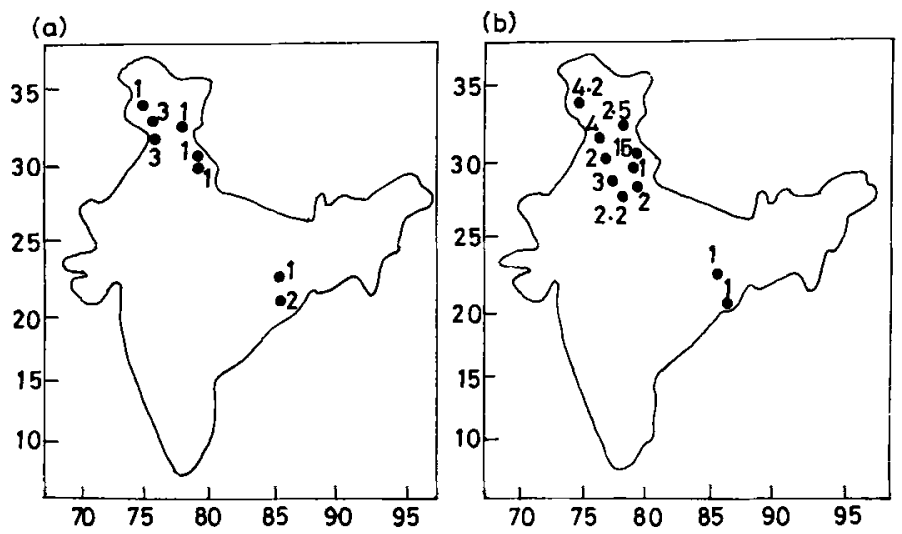

(c)

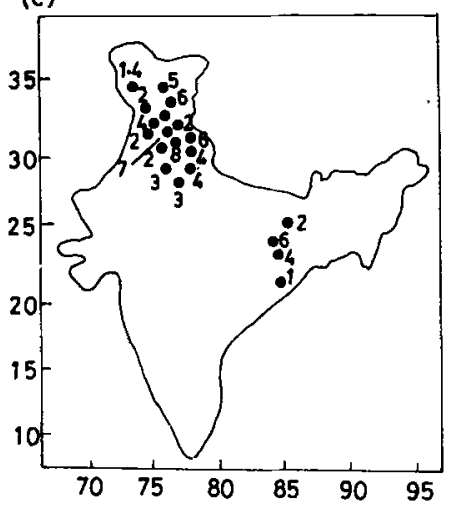

(d)

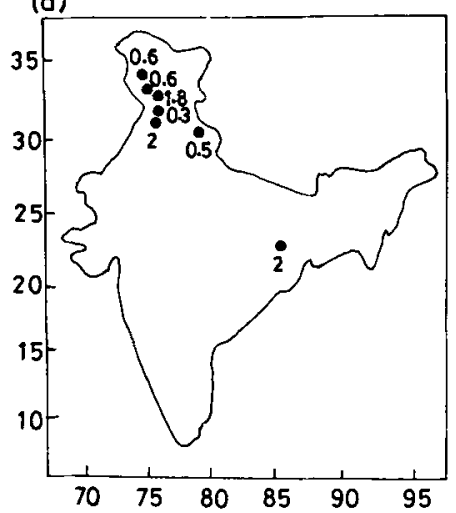

Figure 9. 24 hours accumulated observed rainfall $(\mathrm{cm})$ on (a) 28.2 .1982 (b) 1.3 .1982 (c) 2.3 .1982 and (d) 3.3 .1982 . 
sufficient to accurately predict the rainfall. Cloud-radiation feedback process is also not included in the model. These limitations should be taken into account while judging the simulation obtained from the model. The authors feel that the wind fields and geopotential heights are well predicted up to day 3 . Increasing the resolution and developing sophisticated physical packages can improve the quality of the forecast. It may also be noted that the "high' observed at $700 \mathrm{mb}$ in figures $1 \mathrm{~b}, 4,5 \mathrm{~b}$ and $6 \mathrm{~b}$ are essentially due to the numerical errors introduced by the interpolation of fields from pressure to sigma levels of the model in the vertical and vice versa. The inadequate treatment of orography (Himalayas) in the model might have also contributed to this type of numerical high.

\section{Conclusions}

By using the Global Spectral Model of IIT, Delhi a winter circulation in February 1982 has been successfully simulated. The model has been integrated for five days in Cyber 170/730 computer available in Delhi. The comparison of the forecasted wind and geopotential heights with the respective analyses over the Indian subcontinent shows reasonable agreement. The 24 -hour accumulated precipitation from the model agrees qualitatively only up to two days with the 24-hour rainfall over north India which was under the influence of western disturbance from 27 February 1982 till 3 March 1982.

At present GSM has five sigma levels in the vertical and triangular truncation scheme is used in the horizontal with 21 waves. The resolution of the model in the vertical as well as in the horizontal cannot be increased at present because of the limited core memory of the computer available to the authors. Considering the low resolution of the model, the simulation of the winter circulation patterns over Afghanistan, Pakistan and north India is quite encouraging.

\section{Acknowledgements}

One of the authors (SKD) thanks the scientists of ECMWF and Prof. R P Pearce for their co-operation. Thanks are due to Prof. M P Singh for constant encouragement and support. The authors also thank Dr U C Mohanty for useful discussions and Dr Parashuram and Mr Roy Abraham for help in executing the programmes and preparing the diagrams. One of the authors $(\mathrm{BC})$ thanks CSIR for providing financial assistance in the form of a fellowship during the course of this study.

\section{Appendix}

The $k$ th element of the column vector $Z \downarrow$ is defined as,

$$
Z_{k}=\left(\frac{1}{a(1-\mu 2)} \frac{\partial}{\partial \lambda} P_{v}-\frac{\partial}{a \partial \mu} P_{u}\right)_{k}
$$


where

$$
\begin{aligned}
& P_{u}=\zeta v-\frac{R T_{v}^{\prime}}{a} \frac{\partial}{\partial \lambda} \ln p_{s}-\dot{\sigma} \frac{\partial u}{\partial \sigma} \\
& P_{v}=-\zeta u-\frac{R T_{v}^{\prime}}{a}(1-\mu 2) \frac{\partial}{\partial \mu} \ln p_{s}-\dot{\sigma} \frac{\partial v}{\partial \sigma}
\end{aligned}
$$

The $k$ th element of the column vector $\Upsilon \downarrow$ is defined as,

where

$$
\Upsilon_{k}=\Upsilon_{1 k}+\Upsilon_{2 k}
$$

$$
\begin{aligned}
\Upsilon_{1 k}= & -\frac{1}{a(1-\mu 2)} \frac{\partial}{\partial \lambda}\left(U_{k} \cdot T_{k}^{\prime}\right)-\frac{\partial}{a \partial \mu}\left(V_{k} \cdot T_{k}^{\prime}\right), \\
\Upsilon_{2 k}= & D_{k} \cdot T_{k}^{\prime}-\frac{1}{2 \Delta \sigma_{k}}\left[\dot{\sigma}_{k+\frac{1}{2}}\left(T_{k+1}^{\prime}-T_{k}^{\prime}\right)+\dot{\sigma}_{k-\frac{1}{2}}\left(T_{k}^{\prime}-T_{k-1}^{\prime}\right)\right] \\
& -\frac{1}{2 \Delta \sigma_{k}}\left[( T o _ { k + 1 } - T o _ { k } ) \left\{\sigma_{k+\frac{1}{2}} \sum_{j=1}^{N} \Delta \sigma_{j} \mathbf{V}_{j} \cdot \nabla \ln p_{s}\right.\right. \\
& \left.\left.-\sum_{j=1}^{k} \Delta \sigma_{j} \mathbf{V}_{j} \cdot \nabla \ln p_{s}\right\}\right]-\frac{1}{2 \Delta \sigma_{k}}\left[\left(T o_{k}-T o_{k-1}\right)\right. \\
& \left.\cdot\left\{\sigma_{k-\frac{1}{2}} \sum_{j=1}^{N} \Delta \sigma_{j} \mathbf{V}_{j} \cdot \nabla \ln p_{s}-\sum_{j=1}^{k-1} \Delta \sigma_{j} \mathbf{V}_{j} \cdot \nabla \ln p_{s}\right\}\right] \\
& -\kappa T o_{k} \sum_{j=1}^{N} C k_{j}\left(\mathbf{V}_{j} \cdot \nabla \ln p_{s}\right)-\kappa T^{\prime} v_{k} \sum_{j=1}^{N} C k_{j}\left(\mathbf{V}_{j} \cdot \nabla \ln p_{s}+D_{j}\right) \\
& +\kappa T v_{k}\left(\mathbf{V}_{k} \cdot \nabla \ln p_{s}\right) .
\end{aligned}
$$

The elements of matrix $\tau$ in the thermodynamic equation are defined as,

$$
\begin{aligned}
\tau_{k j}= & \frac{1}{2 \Delta \sigma_{k}}\left[\left(T o_{k+1}-T o_{k}\right)\left\{\sigma_{k+\frac{1}{2}} \Delta \sigma_{j}-\mid \begin{array}{l}
o,(k<j) \\
\Delta \sigma_{j},(k \geqslant j)
\end{array}\right\}\right] \\
& +\frac{1}{2 \Delta \sigma_{k}}\left[\left(T o_{k}-T o_{k-1}\right)\left\{\sigma_{k-\frac{1}{2}} \Delta \sigma_{j}-\mid \begin{array}{l}
o,(k-1<j) \\
\Delta \sigma_{j},(k-1 \geqslant j)
\end{array}\right\}\right] \\
& +\kappa T o_{k} C k_{j} .
\end{aligned}
$$

The $k$ th element of the column vector $Q \downarrow$ is defined as,

$$
\begin{aligned}
Q_{k}= & -\frac{1}{a(1-\mu 2)} \frac{\partial}{\partial \lambda}\left(U_{k} \cdot q_{k}\right)-\frac{1}{a \partial \mu}\left(V_{k} \cdot q_{k}\right)+D_{k} q_{k} \\
& -\frac{1}{2 \Delta \sigma_{k}}\left[\dot{\sigma}_{k+\frac{1}{2}}\left(q_{k+1}-q_{k}\right)+\dot{\sigma}_{k-\frac{1}{2}}\left(q_{k}-q_{k-1}\right)\right] .
\end{aligned}
$$


The $k$ th element of row vector $\boldsymbol{\Pi}$ is defined as,

$$
\Pi_{k} \equiv \Delta \sigma_{k}=\sigma_{k+\frac{1}{2}}-\sigma_{k-\frac{1}{2}}
$$

The hydrostatic matrix $\mathbf{B}$ is upper triangular and its elements are defined as follows:

$$
\begin{aligned}
B_{k l} & =o, l<k, \\
B_{k k} & =\frac{1}{2} \ln \frac{\sigma_{k+\frac{1}{2}}}{\sigma_{k-\frac{1}{2}}}, \quad k \neq 1 \\
& =\ln \frac{\sigma_{1+\frac{1}{2}}}{\sigma_{1}}, \quad k=1, \\
B_{k l} & =\ln \frac{\sigma_{l+\frac{1}{2}}}{\sigma_{l-\frac{1}{2}}}, \quad l>k .
\end{aligned}
$$

The $k$ th element of the column vector $\mathscr{D}$ is defined as,

where

$$
\mathscr{D}_{k}=\left[\frac{1}{a(1-\mu 2)} \frac{\partial}{\partial \lambda} P_{u}+\frac{\partial}{a \partial \mu} P_{v}\right]_{k}-\nabla^{2} E_{k}
$$

Also

$$
E_{k}=\left(U_{k}^{2}+V_{k}^{2}\right) / 2(1-\mu 2) \text {. }
$$

$$
\begin{aligned}
\mathscr{P} & =-\sum_{j=1}^{N} \Delta \sigma_{j}\left(\mathbf{V}_{j} \cdot \nabla \ln p_{s}\right), \\
T_{v} & =T \frac{0.622+q}{0 \cdot 622(1+q)}, \\
\mathscr{R} & =\Phi_{s}+R \mathbf{B} T_{v}(t-\Delta t)+\Delta t R \mathbf{B} \Upsilon_{2}+R T o \ln p_{s}(t-\Delta t)+\Delta t R T o \mathscr{P} .
\end{aligned}
$$

The constant matrix $\mathbf{A} n$ is defined as,

$$
\mathrm{A} n=\frac{a^{2}}{n(n+1)} \mathbf{T}+R(\Delta t)^{2}\{\mathrm{~B} \cdot \tau+T o \downarrow \Pi\}
$$

and $I$ is the unit matrix.

$$
C_{k l}=B_{l k} \frac{\Delta \sigma_{l}}{\Delta \sigma_{k}} ; \text { where matrix } C \text { is lower triangular. }
$$

\section{References}

Baede A P M, Jarraud M and Cubasch U 1979 Adiabatic formulation and organisation of ECMWF's spectral model; Technical Report No. 15, European Centre for Medium range Weather Forecasts, Reading, U.K.

Bourke W 1972 An effective one level primitive equation spectral model; Mon. Weather. Rev. 100 683-689 Burridge D M and Haseler J 1977 A Model for medium range weather forecasting; adiabatic formulation; Technical Report No. 4, European Centre for Medium range Weather Forecasts, Reading, U.K. 
Holloway J L Jr and Manabe A 1971 Simulation of Climate by a global general circulation model. 1. Hydrologic cycle and heat balance; Mon. Weather Rev. 99 335-370

Hoskins B J and Simmons A J 1975 A multi-layer spectral model and the semi-implicit method; Q. J.R. Meteorol. Soc. $101637-655$

Manabe S, Smagorinsky J and Strickler R F 1965 Simulated climatology of a general circulation model with a hydrologic cycle; Mon. Weather Rev. 93 769-798

Manabe S and Strickler R F 1964 On the thermal equilibrium of the atmosphere with convective adjustment; J. Atmos. Sci. 21 361-385

Phillips N A 1957 A coordinate system having some special advantages for numerical forecasting; $J$. Meteorol. 14 184-185

Robert A J 1966 The integration of a lower order spectral form of the primitive meteorological equations; J. Meteorol. Soc. Jpn. 44 237-245

Robert A J 1969 The integration of a spectral model of the atmosphere by the implicit method; Proc. WMO/IUGC Symposium on NWP (Tokyo: Japan Meteorological Agency) pp. VII-19-VII-24

Robert A J, Henderson J and Turnbull C 1972 An implicit time integration scheme for baroclinic models of the atmosphere; Mon. Weather Rev. $100329-335$

Smagorinsky J 1963 General circulation experiments with primitive equations; I. The experiment; Mon. Weather Rev. 91 99-164

Smagorinsky J, Manabe S and Holloway J L Jr 1965 Numerical results from nine-level general circulation model of the atmosphere; Mon. Weather Rev. 93 727-768 